\title{
ON QUASI-INTEGRO DIFFERENTIAL EQUATIONS AND THEIR SOLUTIONS IN $L^{p}$-SPACES
}

\section{SOBHY EL-SAYED IBRAHIM}

Department of Mathematics

College of Basic Education

P. O. Box 34053

Al-Edailiyah

Kuwait

e-mail: sobhyelsayed_55@hotmail.com

\begin{abstract}
In this paper, we consider a general quasi-differential expression $\tau$ of order $n$ with complex coefficients and its formal adjoint $\tau^{+}$in the space $L_{w}^{p}(a, b)$. We show in the case of one singular end-point and under suitable conditions on the function $F(t, y)$ that all solutions of general quasi-integro differential equation $[\tau-\lambda I] y(t)=w F(t, y)(\lambda \in \mathbb{C})$ are in $L_{w}^{p}(a, b) \cap L^{\infty}(a, b)$ for all $\lambda \in \mathbb{C}$ provided that all solutions of the homogeneous differential equations $(\tau-\lambda I) u=0$ and $\left(\tau^{+}-\bar{\lambda} I\right) v=0$ are in $L_{w}^{p}(a, b) \cap L^{\infty}(a, b)$.
\end{abstract}

2010 Mathematics Subject Classification: 34B05, 34B24, 47A10, 47E05.

Keywords and phrases: quasi-differential expressions, regular and singular endpoints, minimal and maximal operators, quasi-differential operators in $L^{p}$-spaces, quasi-integro differential equations and their solutions, boundedness of solutions.

Received September 22, 2016

(C) 2016 Scientific Advances Publishers 


\section{Introduction}

The problem that all solutions of a perturbed linear differential equation belong to $L^{2}(0, b)$ assuming the fact that all solutions of the unperturbed equation possess the same property considered by Wong, Yang and Zettl [14-17]. They showed for an ordinary linear differential equations with real coefficients that, under suitable conditions on the function $F$ that all solutions of the equation

$$
\tau[y]-\lambda w y=w F(t, y) \quad(\lambda \in \mathbb{C}) \text { on }[0, b),
$$

are in $L_{w}^{2}(0, b)$ provided that all those of the equations

$$
(\tau-\lambda I) u=0 \quad(\lambda \in \mathbb{C})
$$

and

$$
\left(\tau^{+}-\bar{\lambda} I\right) v=0 \quad(\lambda \in \mathbb{C})
$$

are in $L_{w}^{2}(0, b)$.

In [7-9], Ibrahim extends their results for a general quasi-differential expression $\tau$ of arbitrary order $n$ with complex coefficients, and considered the property of boundedness of solutions of a general quasiintegro differential equations.

Our objective in this paper is to extend the results in [6-9] and [14-18] to a general quasi-integro differential equations with their solutions in the space $L_{w}^{p}(a, b)$. Also, we show in the case of one singular end-point and under suitable conditions on the integrand function $F$ that all solutions of the general quasi-integro differential equation (1.1) are in $L_{w}^{p}(a, b) \cap L^{\infty}(a, b)$ provided that all solutions of the homogeneous quasiintegro differential equations (1.2) and (1.3) are in $L_{w}^{p}(a, b) \cap L^{\infty}(a, b)$. 


\section{Quasi-differential Operators on $L^{p}$-Spaces}

We deal throughout this paper with a quasi-differential expression of an arbitrary order $n$ defined by a Shin-Zettl matrix in the $L^{p}$-space. The left-hand end-point of the interval $I=[a, b)$ is assumed to be regular but the right-hand end-point may be either regular or singular.

Let $\mathbb{K}$ denote either $\mathbb{R}$, the field of real numbers, or $\mathbb{C}$, the field of complex numbers. For a positive integers $n$ and $m$, let $\mathbb{M}_{n, m}$ denote the vector space of $n \times m$ matrices with $\mathbb{K}$-valued entries and $G L_{m}$ the subset of $\mathbb{M}_{m}:=\mathbb{M}_{m, m}$ consisting of all non-singular matrices. For

$A \in \mathbb{M}_{n, m}$, let $A^{T}$ denote the transpose and $A^{*}$ the adjoint, i.e., the complex conjugate transpose of $A$.

If $A$ is a subset of $\mathbb{M}_{n, n}$ and $I$ is an interval, $B(I, A)$ denotes the set of Lebesgue measurable maps of $I$ into $A$ and $A C_{\text {loc }}(I, A)$ the set of locally absolutely continuous maps. Measurable maps are regarded as equal if they are equal almost everywhere on $I$. Further we define

$$
\begin{aligned}
& L^{p}(I, A):=\left\{\left.y \in B(I, A)|| y\right|^{p} \text { is Lebesgue-integrable }\right\}, \\
&\|y\|_{p, I}:=\left(\int_{I}|y|^{p}\right)^{\frac{1}{p}} \text { for all } y \in L^{p}(I, A) \text { and } p \in[1, \infty), \\
& L^{\infty}(I, A):=\{y \in B(I, A) \mid y \text { is essential bounded }\}, \\
&\|y\|_{\infty, I}:=\operatorname{ess} \sup _{x \in I}|y(x)| \text { for all } y \in L^{\infty}(I, A), \\
& L_{\text {loc }}^{p}(I, A):=\left\{y \in B(I, A)|y| K \in L^{p}(K, A)\right. \text { for all compact } \\
&\text { subinterval } K \text { of } I, p \in[1, \infty)\} .
\end{aligned}
$$


If $r \in[1, \infty)$, then $r^{\prime} \in[1, \infty)$ is always chosen such that $\frac{1}{r}+\frac{1}{r^{\prime}}=1$.

We always assume that $p, q \in[1, \infty)$. If $L^{p}:=\left(I, \mathbb{K}^{s}\right)$ for some positive integer $s$, then $\left(L^{p}\right)^{*}=L^{p^{\prime}}$ for $p \in[1, \infty)$ and $L^{1}$ is a subspace of $\left(L^{\infty}\right)^{*}$, where $(.)^{*}$ denotes the complex conjugate transpose. We refer to [5] for more details.

Let $I$ be an interval with end-points $a, b(-\infty \leq a<b \leq \infty)$, let $n, s$ be positive integers and $p, q \in[1, \infty)$. The quasi-differential expressions are defined in terms of a Shin-Zettl matrix $Z_{n, s}^{p, q}(I)$ on an interval $I$.

Definition 2.1 ([5, 12]). The set $Z_{n, s}^{p, q}(I)$ of Shin-Zettl matrices on $I$ consists of matrices are defined to be the sets of all lower triangular matrices $F=\left\{f_{j, k}\right\}$ of the form

$$
F=\left(\begin{array}{ccc}
f_{0,1} & \cdots & 0 \\
\vdots & \ddots & \vdots \\
f_{n, 1} & \cdots & f_{n, n+1}
\end{array}\right),
$$

whose entries are complex-valued functions on $I$ which satisfy the following conditions:

$$
\begin{aligned}
& f_{0,1} \in L_{\mathrm{loc}}^{p}\left(I, \mathbb{M}_{s}\right) \text { and } f_{n, n+1} \in L_{\mathrm{loc}}^{q^{\prime}}\left(I, \mathbb{M}_{s}\right), \\
& f_{j, k} \in L_{\mathrm{loc}}^{p}\left(I, \mathbb{M}_{s}\right) \text { for all } 1 \leq j \leq n \text { and } 1 \leq k \leq \min \{j+1, n\}, \\
& \qquad f_{j, j+1}(x) \in G L_{s} \text { for all } 0 \leq j \leq n \text { and } x \in I .
\end{aligned}
$$


For $F \in Z_{n, s}^{p, q}(I)$ we define $\widetilde{F}$ as the $(n \times n)$ matrix obtained from $F$ by removing the first row and the last column, i.e.,

$$
\widetilde{F}=\left(\begin{array}{cccc}
f_{1,1} & f_{1,2} & \cdots & 0 \\
\vdots & \vdots & \ddots & 0 \\
f_{n-1,1} & f_{n-1,2} & \cdots & f_{n-1, n} \\
f_{n, 1} & f_{n, 2} & \cdots & f_{n, n}
\end{array}\right) .
$$

Definition 2.2 ([5]). For $\widetilde{F} \in Z_{n, n}^{p, q}(I)$, the quasi-derivatives associated with $\widetilde{F}$ are defined by

$$
\begin{aligned}
& y_{\widetilde{F}}^{[0]}:=y_{\widetilde{F}}, \\
& y_{\widetilde{F}}^{[j]}:=\left(f_{j, j+1}\right)^{-1}\left\{\left(y_{\widetilde{F}}^{[j-1]}\right)^{\prime}-\sum_{k=1}^{j} f_{j, k} y_{\widetilde{F}}^{[k-1]}\right\},(1 \leq j \leq n-1), \\
& y_{\widetilde{F}}^{[n]}:=\left\{\left(y_{\widetilde{F}}^{[n-1]}\right)^{\prime}-\sum_{k=1}^{n} f_{j, k} y_{\widetilde{F}}^{[k-1]}\right\},
\end{aligned}
$$

where the prime ' denotes differentiation.

The quasi-differential expression $\tau_{\widetilde{F}}$ associated with $\widetilde{F}$ is given by:

$$
\tau_{\widetilde{F}}[.]:=i^{n} y_{\widetilde{F}}^{[n]}, \quad(n \geq 2),
$$

this being defined on the set

$$
V\left(\tau_{\widetilde{F}}\right):=\left\{y_{\widetilde{F}}: y_{\widetilde{F}}^{[j-1]} \in A C_{\text {loc }}\left(I, \mathbb{K}^{n}\right), 1 \leq j \leq n\right\},
$$

where $A C_{\text {loc }}\left(I, \mathbb{K}^{n}\right)$, denotes the set of functions which are locally absolutely continuous on every compact subinterval of $I$. 
For $y \in V\left(\tau_{\widetilde{F}}\right)$ we define $Q_{\widetilde{F}} y:=\left(\begin{array}{c}y_{\widetilde{F}}^{[0]} \\ \vdots \\ y_{\widetilde{F}}^{[n-1]}\end{array}\right)$.

Clearly the maps $\tau_{\widetilde{F}}: V\left(\tau_{\widetilde{F}}\right) \rightarrow B\left(I, \mathbb{K}^{n}\right)$ and $Q_{\widetilde{F}}: V\left(\tau_{\widetilde{F}}\right) \rightarrow A C_{\text {loc }}\left(I, \mathbb{K}^{n}\right)$ are linear.

In analogy to the adjoint and the transpose of a matrix, there are two different "(formal) adjoint" of a quasi-differential expression, we refer to [2-5] and [7-10] for more details.

In the following, we always assume that $\widetilde{F} \in Z_{n, n}^{p, q}$ and $\tau_{\widetilde{F}}:=\tau_{p, q}$. The formal adjoint $\tau_{p, q}^{+}$of $\tau_{p, q}$ is defined by the matrix $\widetilde{F}^{+}$given by

$$
\widetilde{F}^{+}=-J_{n}^{-1} \widetilde{F}^{*} J_{n}
$$

where $\widetilde{F}^{*}$ is the conjugate transpose of $\widetilde{F}$ and $J_{n}$ is the non-singular $(n \times n)$ matrix

$$
J_{n}=\left((-1)^{j} \delta_{j, n+1-k}\right)_{\substack{1 \leq j \leq n \\ 1 \leq k \leq n}}
$$

$\delta$ being the Kronecker delta. If $\widetilde{F}^{+}=f_{j, k}^{+}$, then it follows that

$$
f_{j, k}^{+}=(-1)^{j+k+1} \bar{f}_{n-k+1, n-j+1} \cdot
$$

The quasi-derivatives associated with the matrix $\widetilde{F}^{+}$in $Z_{n, n}^{p, q}(I)$ are therefore

$$
\begin{aligned}
& y_{+}^{[0]}:=y, \\
& y_{+}^{[j]}:=\left(\bar{f}_{n-j, n-j+1}\right)^{-1}\left\{\left(y_{+}^{[j-1]}\right)^{\prime}-\sum_{k=1}^{j}(-1)^{j+k+1} \bar{f}_{n-k+1, n-j+1} y_{+}^{[k-1]}\right\},
\end{aligned}
$$




$$
\begin{gathered}
y_{+}^{[n]}:=\left\{\left(y_{+}^{[n-1]}\right)^{\prime}-\sum_{k=1}^{n}(-1)^{n+k+1} \bar{f}_{n-k+1,1} y_{+}^{[k-1]}\right\}, \\
\tau_{q^{\prime}, p^{\prime}}^{+}[.]:=i^{n} y_{+}^{[n]}(n \geq 2) \text { for all } y \in V\left(\tau_{q^{\prime}, p^{\prime}}^{+}\right), \\
V\left(\tau_{q^{\prime}, p^{\prime}}^{+}\right):=\left\{y: y_{+}^{[j-1]} \in A C_{\mathrm{loc}}\left(I, \mathbb{K}^{n}\right), 1 \leq j \leq n\right\},
\end{gathered}
$$

Note that. $\left(\widetilde{F}^{+}\right)^{+}=\widetilde{F}$ and so $\left(\tau_{q^{\prime}, p^{\prime}}^{+}\right)^{+}=\tau_{p, q}$. We refer to [2-5], [7-10], and [19,20] for a full account of the above and subsequent results on quasi-differential expressions.

For $u \in V\left(\tau_{p, q}\right), v \in V\left(\tau_{q^{\prime}, p^{\prime}}^{+}\right)$and $\alpha, \beta \in I$, we have Green's formula,

$$
\int_{\alpha}^{\beta}\left\{\bar{v} \tau_{p, q}[u]-u \overline{\tau_{q^{\prime}, p^{\prime}}^{+}[v]}\right\} d x=[u, v](\beta)-[u, v](\alpha),
$$

where

$$
\begin{aligned}
{[u, v](x) } & =i^{n}\left(\sum_{r=0}^{n-1}(-1)^{r+n+1} u^{[r]} \overline{v_{+}^{[n-r-1]}}\right)(x) \\
& =(-i)^{n}\left(Q_{\widetilde{F}}^{T} u J_{n \times n} Q_{\widetilde{F}} \bar{v}\right)(x) . \\
& =(-i)^{n}\left(u, u^{[1]}, \ldots, u^{[n-1]}\right) J_{n \times n}\left(\begin{array}{c}
\bar{v} \\
\vdots \\
\bar{v}_{+}^{[n-1]}
\end{array}\right)(x),
\end{aligned}
$$

see [2-5], [7-10], and [19]. Let $w: I \rightarrow \mathbb{R}$ be a non-negative weight function with $w \in L_{\mathrm{loc}}^{1}(I)$ and $w>0$ (for almost all $x \in I$ ). Then $H^{r}=L_{w}^{r}\left(I, \mathbb{K}^{n}\right)$ denotes the Hilbert function space of equivalence classes of Lebesgue measurable functions such that

$$
\|y\|_{r, I}:=\left(\int_{I}|y|^{r} w\right)^{\frac{1}{r}} \text { for all } y \in L^{r}(I, \widetilde{F}) \text { and } r \in[1, \infty) .
$$


The equation

$$
\tau_{p, q}[u]-\lambda w u=0 \quad(\lambda \in \mathbb{C}) \text { on } \quad I,
$$

is said to be regular at the left end-point $a \in \mathbb{R}$, if for all $X \in[a, b)$,

$$
a \in \mathbb{R}, w, f_{j, k} \in L^{1}[a, X], \quad j, k=1,2, \ldots, n,
$$

otherwise (2.12) is said to be singular at $a$. If (2.12) is regular at both end-points, then it is said to be regular; in this case we have,

$$
a, b \in \mathbb{R}, w, f_{j, k} \in L^{1}[a, b], \quad j, k=1,2, \ldots, n .
$$

We shall be concerned with the case when $a$ is a regular end-point of the Equation (2.13), the end-point $b$ being allowed to be either regular or singular. Note that, in view of (2.6), an end-point of $I$ is regular for (2.13), if and only if it is regular for the equation

$$
\tau_{p, q}^{+}[v]-\bar{\lambda} w v=0 \quad(\lambda \in \mathbb{C}) \text { on } I .
$$

Definition 2.3 ([5, 12]). (a) The maximal operators corresponding to $\tau_{p, q}, \tau_{q^{\prime}, p^{\prime}}^{+}$are defined as operators from a subspaces of $L_{w}^{p}$ into $L_{w}^{q}, p, q$ are arbitrary.

$$
\begin{aligned}
& T\left(\tau_{p, q}\right):=w^{-1} \tau[u] \text { for all } u \in D\left[T\left(\tau_{p, q}\right)\right], \\
& D\left[T\left(\tau_{p, q}\right)\right]:=\left\{u \in V\left(\tau_{p, q}\right) \cap L_{w}^{p} \mid w^{-1} \tau_{p, q}[u] \in L_{w}^{q}\right\}, \\
& T\left(\tau_{q^{\prime}, p^{\prime}}^{+}\right):=w^{-1} \tau^{+}[v] \text { for all } v \in D\left[T\left(\tau_{q^{\prime}, p^{\prime}}^{+}\right)\right], \\
& D\left[T\left(\tau_{q^{\prime}, p^{\prime}}^{+}\right)\right]:=\left\{v \in V\left(\tau_{q^{\prime}, p^{\prime}}^{+}\right) \cap L_{w}^{p} \mid w^{-1} \tau_{q^{\prime}, p^{\prime}}^{+}[v] \in L_{w}^{q}\right\} .
\end{aligned}
$$

The subspaces $D\left[T\left(\tau_{p, q}\right)\right]$ and $D\left[T\left(\tau_{q^{\prime}, p^{\prime}}^{+}\right)\right]$of $L_{w}^{p}\left(I, \mathbb{K}^{n}\right)$ are the domains of the so-called maximal operators $T\left(\tau_{p, q}\right)$ and $T\left(\tau_{p^{\prime}, q^{\prime}}^{+}\right)$, respectively. 
(b) For the regular problem the minimal operators $T_{0}\left(\tau_{p, q}\right)$ and $T_{0}\left(\tau_{q^{\prime}, p^{\prime}}^{+}\right)$are the restrictions of $w^{-1} \tau_{p, q}[u]$ and $w^{-1} \tau_{q^{\prime}, p^{\prime}}^{+}[v]$ to the subspaces:

$$
\left.\begin{array}{l}
D_{0}\left(\tau_{p, q}\right):=\left\{u: u \in D\left(\tau_{p, q}\right), \quad\left(Q_{\widetilde{F}} u\right)(a)=\left(Q_{\widetilde{F}} u\right)(b)=0\right\} \\
D_{0}\left(\tau_{q^{\prime}, p^{\prime}}^{+}\right):=\left\{v: v \in D\left(\tau_{q^{\prime}, p^{\prime}}^{+}\right),\left(Q_{\widetilde{F}} v_{+}\right)(a)=\left(Q_{\widetilde{F}} v_{+}\right)(b)=0\right\}
\end{array}\right\} .
$$

The subspaces $D_{0}\left(\tau_{p, q}\right)$ and $D_{0}\left(\tau_{q^{\prime}, p^{\prime}}^{+}\right)$are dense in $L_{w}^{p}\left(I, \mathbb{K}^{n}\right)$ and $T_{0}\left(\tau_{p, q}\right)$ and $T_{0}\left(\tau_{q^{\prime}, p^{\prime}}^{+}\right)$are closed operators (see [2], [5], [8-12], and [20]).

In the singular problem, we first introduce the operators $T_{0}^{\prime}\left(\tau_{p, q}\right)$ and $T_{0}^{\prime}\left(\tau_{q^{\prime}, p^{\prime}}^{+}\right) ; T_{0}^{\prime}\left(\tau_{p, q}\right)$ being the restriction of $w^{-1} \tau_{p, q}[$.$] to the subspace$

$$
D_{0}^{\prime}\left(\tau_{p, q}\right):=\left\{u: u \in D\left(\tau_{p, q}\right) \mid \operatorname{supp}(u)\right. \text { is compact }
$$

support in the interior of $I\}$,

and with $T_{0}^{\prime}\left(\tau_{q^{\prime}, p^{\prime}}^{+}\right)$defined similarly. These operators are densely defined and closable in $L_{w}^{p}\left(I, \mathbb{K}^{n}\right)$; and we define the minimal operators $T_{0}\left(\tau_{p, q}\right)$ and $T_{0}\left(\tau_{q^{\prime}, p^{\prime}}^{+}\right)$to be their respective closures (see [2-5], [7-10], and [19]). We denote the domains of $T_{0}\left(\tau_{p, q}\right)$ and $T_{0}\left(\tau_{q^{\prime}, p^{\prime}}^{+}\right)$by $D_{0}\left(\tau_{p, q}\right)$ and $D_{0}\left(\tau_{q^{\prime}, p^{\prime}}^{+}\right)$, respectively. It can be shown that

$$
\left.\begin{array}{c}
u \in D_{0}\left(\tau_{p, q}\right) \Rightarrow\left(Q_{\widetilde{F}} u\right)(a)=0 \\
v \in D_{0}\left(\tau_{p^{\prime}, q^{\prime}}^{+}\right) \Rightarrow\left(Q_{\widetilde{F}} v_{+}\right)(a)=0
\end{array}\right\},
$$

because we are assuming that $a$ is a regular end-point. Clearly $T_{0}\left(\tau_{p, q}\right)$ and $T\left(\tau_{p, q}\right)$ are linear operators of $L_{w}^{p}$ into $L_{w}^{q}$ and $T_{0}\left(\tau_{p, q}\right) \subset T\left(\tau_{p, q}\right)$.

Moreover, in both regular and singular problems, we have

$$
T_{0}^{*}\left(\tau_{p, q}\right)=T\left(\tau_{q^{\prime}, p^{\prime}}^{+}\right), \quad T^{*}\left(\tau_{p, q}\right)=T_{0}\left(\tau_{q^{\prime}, p^{\prime}}^{+}\right),
$$


see [2-10] and [20, Section 5] in the case when $\tau_{p, q}=\tau_{q^{\prime}, p^{\prime}}^{+}$and compare with treatment in [2, Section III.10.3] in general case. Also, we refer to [11-14] and [17-19] for more details.

\section{3. $L_{w}^{p}$-Solutions}

In this section, we shall concerned with $L_{w}^{p}$-solutions of the quasiintegro differential equations, and we denote for $\tau_{p, q}$ by $\tau$ and $\tau_{p, q}^{+}$by $\tau^{+}$. Denote by $S(\tau)$ and $S\left(\tau^{+}\right)$the sets of all solutions of the equations

$$
\left[\tau-\lambda_{0} I\right] u=0 \quad\left(\lambda_{0} \in \mathbb{C}\right),
$$

and

$$
\left[\tau^{+}-\overline{\lambda_{0}} I\right] v=0 \quad\left(\lambda_{0} \in \mathbb{C}\right)
$$

respectively. Let $\varphi_{j}(t, \lambda), j=1,2, \ldots, n$ be the solutions of the homogeneous equation $[\tau-\lambda I] u=0(\lambda \in \mathbb{C})$ satisfying:

$$
\varphi_{j}^{[k-1]}\left(t_{0}, \lambda\right)=\delta_{k, r+1} \text { for all } t_{0} \in[a, b), \quad(j, k=1,2, \ldots n),
$$

for fixed $t_{0}, a<t_{0}<b$. Then $\varphi_{j}(t, \lambda)$ is continuous in $(t, \lambda)$ for $0<t<b,|\lambda|<\infty$, and for fixed $t$ it is entire in $\lambda$. Let $\varphi_{k}^{+}(t, \lambda)$, $k=1,2, \ldots, n$ denote the solutions of the adjoint homogeneous equation $\left[\tau^{+}-\bar{\lambda} I\right] v=0(\lambda \in \mathbb{C})$ satisfying:

$$
\left(\varphi_{k}^{+}\right)^{[r]}\left(t_{0}, \lambda\right)=(-1)^{k+r} \delta_{k, n-r} \text { for all } t_{0} \in[0, b), \quad(k=1,2, \ldots, n) .
$$

Suppose $a<c<b$, by [3], [7-9], and [12-16], a solution of the equation

$$
[\tau-\lambda I] u=w f \quad(\lambda \in \mathbb{C}), \quad f \in L_{w}^{1}(a, b)
$$

satisfying $u(c)=0$ is giving by

$$
\varphi(t, \lambda)=\left(\frac{\left(\lambda-\lambda_{0}\right)}{i^{n}}\right) \sum_{j, k=1}^{n} \xi^{j k} \varphi_{j}(t, \lambda) \int_{a}^{t} \overline{\varphi_{k}^{+}(s, \lambda)} f(s) w(s) d s
$$


where $\varphi_{k}^{+}(t, \lambda)$ stands for the complex conjugate of $\varphi_{k}(t, \lambda)$ and for each $j, k, \xi^{j k}$ is constant which is independent of $t, \lambda$ (but does depend in general on $\left.t_{0}\right)$.

The next lemma is a form of the variation of parameters formula for a general quasi-differential equation is giving by the following lemma:

Lemma 3.1. Suppose $f \in L_{w}^{1}(0, b)$ locally integrable function and $\varphi(t, \lambda)$ is the solution of the Equation (3.3) satisfying:

$$
\varphi^{[r]}\left(t_{0}, \lambda\right)=\alpha_{r+1} \quad \text { for } \quad r=0,1, \ldots, n-1, t_{0} \in[a, b) .
$$

Then

$$
\begin{aligned}
\varphi(t, \lambda)=\sum_{j=1}^{n} \alpha_{j}(\lambda) \varphi_{j}\left(t, \lambda_{0}\right) & +\left(\left(\lambda-\lambda_{0}\right) / i^{n}\right) \sum_{j, k=1}^{n} \xi^{j k} \varphi_{j}\left(t, \lambda_{0}\right) \\
& \times \int_{a}^{t} \overline{\varphi_{k}^{+}\left(s, \lambda_{0}\right)} f(s) w(s) d s
\end{aligned}
$$

for some constants $\alpha_{1}(\lambda), \alpha_{2}(\lambda), \ldots, \alpha_{n}(\lambda) \in \mathbb{C}$, where $\varphi_{j}\left(t, \lambda_{0}\right)$ and $\varphi_{k}^{+}\left(t, \lambda_{0}\right), j, k=1,2, \ldots, n$ are solutions of the Equations (3.1) and (3.2), respectively, $\xi^{j k}$ is a constant which is independent of $t$.

Lemma 3.2 ([13], Gronwall's inequality). Let $u(t)$ and $v(t)$ be two real-valued functions defined, non-negative and $u, v \in L^{1}\left(t_{0}, t\right)$ for $t>t_{0}$, and if

$$
u(t) \leq c+\int_{t_{0}}^{t} u(s) v(s) d s, \quad c>0
$$

for some positive constant $c$, then

$$
u(t) \leq c \exp \left(\int_{0}^{t} v(s) d s\right) d s .
$$


Lemma 3.3. Suppose that for some $\lambda_{0} \in \mathbb{C}$ all solutions of the Equations (3.1) and (3.2) are in $L_{w}^{2}(a, b)$. Then all solutions of the Equations (1.2) and (1.3) are in $L_{w}^{2}(a, b)$ for every complex number $\lambda \in \mathbb{C}$.

Proof. The proof is similar to that in [8, Lemma 3.5].

Lemma 3.4. If all solutions of the equation $\left[\tau-\lambda_{0} w\right] u=0$ are bounded on $[a, b)$ and $\varphi_{k}^{+}\left(t, \lambda_{0}\right) \in L_{w}^{1}(a, b)$ for some $\lambda_{0} \in \mathbb{C}, k=1, \ldots, n$. Then all solutions of the equation $[\tau-\lambda w] u=0$ are also bounded on $[a, b)$ for every complex number $\lambda \in \mathbb{C}$.

Lemma 3.5. Suppose that for some complex number $\lambda_{0} \in \mathbb{C}$ all solutions of the Equation (3.1) are in $L_{w}^{p}(a, b)$ and all solutions of (3.2) are in $L_{w}^{q}(a, b)$. Suppose $f \in L_{w}^{p}(a, b)$, then all solutions of the Equation (3.3) are in $L_{w}^{p}(a, b)$ for all $\lambda \in \mathbb{C}$.

Proof. Let $\left\{\varphi_{1}\left(t, \lambda_{0}\right), \ldots, \varphi_{n}\left(t, \lambda_{0}\right)\right\},\left\{\varphi_{1}^{+}\left(s, \lambda_{0}\right), \ldots, \varphi_{n}^{+}\left(s, \lambda_{0}\right)\right\}$ be two sets of linearly independent solutions of the Equations (3.1) and (3.2), respectively. Then for any solutions $\varphi(t, \lambda)$ of the equation $[\tau-\lambda I] \varphi=w f$ $(\lambda \in \mathbb{C})$ which may be written as follows

$$
\left[\tau-\lambda_{0} w\right] \varphi=\left(\lambda-\lambda_{0}\right) w \varphi+w f
$$

and it follows from (3.4) that

$$
\begin{aligned}
\varphi(t, \lambda)= & \sum_{j=1}^{n} \alpha_{j}(\lambda) \varphi_{j}\left(t, \lambda_{0}\right)+\frac{1}{i^{n}} \sum_{j, k=1}^{n} \xi^{j k} \varphi_{j}\left(t, \lambda_{0}\right) \\
& \times \int_{a}^{t} \overline{\varphi_{k}^{+}\left(t, \lambda_{0}\right)}\left[\left(\lambda-\lambda_{0}\right) \varphi(s, \lambda)+f(s)\right] w(s) d s,
\end{aligned}
$$


for some constants $\alpha_{1}(\lambda), \alpha_{2}(\lambda), \ldots, \alpha_{n}(\lambda) \in \mathbb{C}$. Hence

$$
\begin{aligned}
|\varphi(t, \lambda)| \leq & \sum_{j=1}^{n}\left(\left|\alpha_{j}(\lambda)\right|\left|\varphi_{j}\left(t, \lambda_{0}\right)\right|\right)+\sum_{j, k=1}^{n}\left|\xi^{j k}\right|\left|\varphi_{j}\left(t, \lambda_{0}\right)\right| \\
& \times \int_{a}^{t} \overline{\varphi_{k}^{+}\left(t, \lambda_{0}\right)}\left[\left|\lambda-\lambda_{0}\right||\varphi(s, \lambda)|+|f(s)|\right] w(s) d s .
\end{aligned}
$$

Since $f \in L_{w}^{p}(a, b)$ and $\varphi_{k}^{+}\left(., \lambda_{0}\right) \in L_{w}^{q}(a, b)$ for some $\lambda_{0} \in \mathbb{C}$, then $\varphi_{k}^{+}\left(., \lambda_{0}\right) f \in L_{w}^{1}(a, b)$ for some $\lambda_{0} \in \mathbb{C}$ and $k=1, \ldots, n$. Setting

$$
C_{j}(\lambda)=\sum_{j, k=1}^{n}\left|\xi^{j k}\right| \int_{a}^{b}\left|\overline{\varphi_{k}^{+}\left(s, \lambda_{0}\right)}\right| f(s) \mid w(s) d s, \quad j=1,2, \ldots, n,
$$

then

$$
\begin{aligned}
|\varphi(t, \lambda)| \leq & \sum_{j=1}^{n}\left(\left|\alpha_{j}(\lambda)\right|+C_{j}(\lambda)\right)\left|\varphi_{j}\left(t, \lambda_{0}\right)\right|+\left|\lambda-\lambda_{0}\right| \\
& \times \sum_{j, k=1}^{n}\left|\xi^{j k}\right|\left|\varphi_{j}\left(t, \lambda_{0}\right)\right| \int_{a}^{b}\left|\overline{\varphi_{k}^{+}\left(s, \lambda_{0}\right)}\right||\varphi(s, \lambda)| w(s) d s .
\end{aligned}
$$

On application of the Cauchy-Schwartz inequality to the integral in (3.9), we get

$$
\begin{aligned}
|\varphi(t, \lambda)| \leq & \sum_{j=1}^{n}\left(\left|\alpha_{j}(\lambda)\right|+C_{j}(\lambda)\right)\left|\varphi_{j}\left(t, \lambda_{0}\right)\right|+\left|\lambda-\lambda_{0}\right| \sum_{j, k=1}^{n}\left|\xi^{j k}\right|\left|\varphi_{j}\left(t, \lambda_{0}\right)\right| \\
& \times\left(\int_{a}^{b}\left|\overline{\varphi_{k}^{+}\left(t, \lambda_{0}\right)}\right|^{q} w(s) d s\right)^{\frac{1}{q}}\left(\int_{a}^{b}|\varphi(s, \lambda)|^{p} w(s) d s\right)^{\frac{1}{p}}
\end{aligned}
$$

From the inequality $(u+v)^{p} \leq 2^{(p-1)}\left(u^{p}+v^{p}\right)$, it follows that

$$
\begin{aligned}
|\varphi(t, \lambda)|^{p} \leq & 2^{2(p-1)} \sum_{j=1}^{n}\left(\left|\alpha_{j}(\lambda)\right|+C_{j}(\lambda)\right)^{p}\left|\varphi_{j}\left(t, \lambda_{0}\right)\right|^{p} \\
& +2^{2(p-1)}\left|\lambda-\lambda_{0}\right|^{p} \sum_{j, k=1}^{n}\left|\xi^{j k}\right|^{p}\left|\varphi_{j}\left(t, \lambda_{0}\right)\right|^{p} \\
& \times\left(\int_{a}^{b}\left|\overline{\varphi_{k}^{+}\left(t, \lambda_{0}\right)}\right|^{q} w(s) d s\right)^{\frac{p}{q}}\left(\int_{a}^{b}|\varphi(s, \lambda)|^{p} w(s) d s\right) .
\end{aligned}
$$


By hypothesis, there exist positive constant $K_{0}$ and $K_{1}$ such that

$$
\left\|\varphi_{j}\left(t, \lambda_{0}\right)\right\|_{L_{w}^{p}(a, b)} \leq K_{0} \text { and }\left\|\overline{\varphi_{k}^{+}\left(s, \lambda_{0}\right)}\right\|_{L_{w}^{q}(a, b)} \leq K_{1},
$$

$j, k=1,2, \ldots, n$. Hence

$$
\begin{aligned}
|\varphi(t, \lambda)|^{p} \leq & 2^{2(p-1)} \sum_{j=1}^{n}\left(\left|\alpha_{j}(\lambda)\right|+C_{j}(\lambda)\right)^{p}\left|\varphi_{j}\left(t, \lambda_{0}\right)\right|^{p} \\
& +2^{2(p-1)} K_{1}^{p}\left|\lambda-\lambda_{0}\right|^{p} \sum_{j, k=1}^{n}\left|\xi^{j k}\right|^{p}\left|\varphi_{j}\left(t, \lambda_{0}\right)\right|^{p} \\
& \times\left(\int_{a}^{b}|\varphi(s, \lambda)|^{p} w(s) d s\right) .
\end{aligned}
$$

Integrating the inequality in (3.13) between $a$ and $t$, we obtain

$$
\begin{aligned}
\int_{0}^{t}|\varphi(s, \lambda)|^{p} w(s) d s \leq & K_{2}+\left(2^{2(p-1)}\left|\lambda-\lambda_{0}\right|^{p} \sum_{j, k=1}^{n}\left|\xi^{j k}\right|^{p}\right) \\
& \times \int_{a}^{t}\left|\varphi_{j}\left(t, \lambda_{0}\right)\right|^{p}\left(\int_{a}^{s}|\varphi(x, \lambda)|^{p} w(x) d x\right) w(s) d s,
\end{aligned}
$$

where

$$
K_{2}=2^{2(p-1)} K_{0}^{p} \sum_{j=1}^{n}\left(\left|\alpha_{j}(\lambda)\right|+C_{j}(\lambda)\right)^{p}
$$

Now, on using Gronwall's inequality, it follows that

$$
\begin{aligned}
& \int_{0}^{t}|\varphi(s, \lambda)|^{p} w(s) d s \\
& \quad \leq K_{2} \exp \left(2^{2(p-1)} K_{1}^{p}\left|\lambda-\lambda_{0}\right|^{p} \sum_{j, k=1}^{n}\left|\xi^{j k}\right|^{p} \int_{a}^{t}\left|\varphi_{j}\left(t, \lambda_{0}\right)\right|^{p} w(s) d s\right)
\end{aligned}
$$

Since, $\varphi_{j}\left(t, \lambda_{0}\right) \in L_{w}^{p}(a, b)$ for some $\lambda_{0} \in \mathbb{C}$ and for $j=1, \ldots, n$, then $\varphi(t, \lambda) \in L_{w}^{p}(0, b)$ for all $\lambda \in \mathbb{C}$.

Remark. Lemma 3.5 also holds if the function $f$ is bounded on $[a, b)$. 
Lemma 3.6. Let $f \in L_{w}^{p}(0, b)$. Suppose for some $\lambda_{0} \in \mathbb{C}$ that:

(i) All solutions of $\left(\tau^{+}-\bar{\lambda} I\right) \varphi^{+}=0$ are in $L_{w}^{q}(a, b)$.

(ii) $\varphi_{j}\left(t, \lambda_{0}\right), j=1, \ldots, n$ are bounded on $[0, b)$.

Then all solutions $\varphi(t, \lambda)$ of the Equation (3.4) are in $L_{w}^{p}(a, b)$ for all $\lambda \in \mathbb{C}$.

\section{4. $L_{w}^{p}$-Boundedness}

In this section, we shall consider the question of determining conditions under which all solutions of the Equation (1.1) are in $L_{w}^{p}(a, b) \cap L^{\infty}(a, b)$.

Suppose there exist non-negative continuous functions $k(t)$ and $h(t)$ on $[a, b), a<b \leq \infty$ such that the function $F(t, y)$ in (1.1) satisfies:

$$
|F(t, y)| \leq k(t)+h(t)|y(t)|^{\sigma} \text { for } t \geq 0,-\infty<y(t)<\infty,
$$

for some $\sigma \in[0,1]$; see [1], [8], and [18-19].

In the sequel, we shall require the following nonlinear integral inequality which generalizes those integral inequalities used in [1], [7-9], and [13-18].

Lemma 4.1 (cf. [8, 17]). Let $u(t)$ and $v(t)$ be two non-negative functions, locally integrable on the interval $I=[a, b)$. Then the inequality

$$
u(t) \leq c+\int_{0}^{t} v(s) u^{\sigma}(s) d x, c>0
$$

for $0 \leq \sigma<1$, implies that

$$
\left.u(t) \leq(c)^{(1-\sigma)}+(1-\sigma) \int_{0}^{t} v(s) d s\right)^{\frac{1}{(1-\sigma)}} d s
$$

In particular, if $v(s) \in L^{1}(a, b)$, then (4.2) implies that $u(t)$ is bounded. 
Theorem 4.2. Suppose that $F$ satisfies (4.1) with $\sigma=1$, and that

(i) $S(\tau) \cup S\left(\tau^{+}\right) \subset L^{\infty}(0, b)$ for some $\lambda_{0} \in \mathbb{C}$,

(ii) $k(t)$ and $h(t) \in L_{w}^{1}(0, b)$ for all $t \in[a . b)$.

Then all solutions $\varphi(t, \lambda)$ of the Equation (1.1) are bounded on $[a, b)$ for all $\lambda \in \mathbb{C}$.

Proof. Note that (4.1) and Lemma 3.6 implies that all solutions are defined on [a,b); see [2, Chapter 3], [7-9], and [13] and let $\left\{\varphi_{1}\left(t, \lambda_{0}\right)\right.$, $\left.\varphi_{2}\left(t, \lambda_{0}\right), \ldots, \varphi_{n}\left(t, \lambda_{0}\right)\right\},\left\{\varphi_{1}^{+}\left(s, \lambda_{0}\right),\left\{\varphi_{2}^{+}\left(s, \lambda_{0}\right), \ldots, \varphi_{n}^{+}\left(s, \lambda_{0}\right)\right\}\right.$ be two sets of linearly independent solutions of the Equations (3.1) and (3.2), respectively, and let $\varphi(t, \lambda)$ be any solution of $(1.1)$ on $[a, b)$, then by Lemma 3.1, we have

$$
\begin{aligned}
\varphi(t, \lambda)= & \sum_{j=1}^{n} \alpha_{j}(\lambda) \varphi_{j}\left(t, \lambda_{0}\right)+\frac{1}{i^{n}}\left(\lambda-\lambda_{0}\right) \sum_{j, k=1}^{n} \xi^{j k} \varphi_{j}\left(t, \lambda_{0}\right) \\
& \times \int_{a}^{t} \overline{\varphi_{k}^{+}\left(s, \lambda_{0}\right)} F(s, y) w(s) d s .
\end{aligned}
$$

Hence,

$$
\begin{aligned}
|\varphi(t, \lambda)| \leq & \sum_{j=1}^{n}\left|\alpha_{j}(\lambda)\right|\left|\varphi_{j}\left(t, \lambda_{0}\right)\right|+\left|\lambda-\lambda_{0}\right| \sum_{j, k=1}^{n}\left|\xi^{j k}\right|\left|\varphi_{j}\left(t, \lambda_{0}\right)\right| \\
& \times \int_{a}^{t}\left|\varphi_{k}^{+}\left(s, \lambda_{0}\right)\right|\left(k(s)+h(s)\left|\varphi_{j}(s, \lambda)\right|\right) w(s) d s .
\end{aligned}
$$

Since $k(s) \in L_{w}^{1}(a, b)$ and $\varphi_{k}^{+}\left(s, \lambda_{0}\right) \in L_{w}^{\infty}(a, b), k=1,2, \ldots, n$ for some $\lambda_{0} \in \mathbb{C}$, we have $\varphi_{k}^{+}\left(s, \lambda_{0}\right) k(s) \in L_{w}^{1}(a, b)$ for some $\lambda_{0} \in \mathbb{C}$. Setting

$$
C_{j}=\left|\lambda-\lambda_{0}\right| \sum_{j, k=1}^{n}\left|\xi^{j k}\right| \int_{a}^{t}\left|\overline{\varphi_{k}^{+}\left(s, \lambda_{0}\right)}\right| k(s) w(s) d s, \quad j=1,2, \ldots, n .
$$


Then

$$
\begin{aligned}
|\varphi(t, \lambda)| \leq & \sum_{j=1}^{n}\left(C_{j}+\left|\alpha_{j}(\lambda)\right|\right)\left|\varphi_{j}\left(t, \lambda_{0}\right)\right| \\
& +\left|\lambda-\lambda_{0}\right| \sum_{j, k=1}^{n}\left|\xi^{j k}\right|\left|\varphi_{j}\left(t, \lambda_{0}\right)\right| \\
& \times \int_{0}^{t}\left|\overline{\varphi_{k}^{+}\left(t, \lambda_{0}\right)}\right| h(s)|\varphi(s, \lambda)| w(s) d s .
\end{aligned}
$$

By hypothesis, there exist a positive constants $K_{0}$ and $K_{1}$ such that

$$
\left|\varphi_{j}\left(t, \lambda_{0}\right)\right| \leq K_{0} \text { and }\left|\overline{\varphi_{k}^{+}\left(t, \lambda_{0}\right)}\right| \leq K_{1} \text { for all } t \in[0, b)
$$

$j, k=1,2, \ldots, n$. Hence,

$$
\begin{aligned}
|\varphi(t, \lambda)| \leq & K_{0} \sum_{j=1}^{n}\left(C_{j}+\left|\alpha_{j}(\lambda)\right|\right)+K_{0} K_{1}\left|\lambda-\lambda_{0}\right| \\
& \times \sum_{j, k=1}^{n}\left|\xi^{j k}\right| \int_{0}^{t} h(s)|\varphi(s, \lambda)| w(s) d s .
\end{aligned}
$$

Applying Gronwall's inequality to (4.6) and using (ii), we deduce that $|\varphi(t, \lambda)|$ is finite and hence the result.

Theorem 4.3. Suppose that $F$ satisfies (4.1) with $\sigma=1$, and that

(i) $S(\tau) \cup S\left(\tau^{+}\right) \subset L_{w}^{\infty}(a, b)$ for some $\lambda_{0} \in \mathbb{C}$,

(ii) $k(t)$ and $h(t) \in L_{w}^{q}(a, b)$ for all $t \in[a . b)$.

Then all solutions $\varphi(t, \lambda)$ of the Equation (1.1) are in $L_{w}^{p}(a, b)$ for all $\lambda \in \mathbb{C}$. 
Proof. The proof follows on applying the Cauchy-Schwartz inequality for the integral in (4.5) as:

$$
\begin{aligned}
& \int_{a}^{t}\left|\overline{\varphi_{k}^{+}\left(t, \lambda_{0}\right)}\right||h(s)||\varphi(s, \lambda)| w(s) d s
\end{aligned}
$$

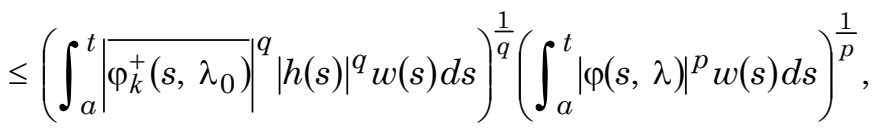

and hence the result. We refer to [1] and [16] for more details.

Corollary 4.4. Suppose that $|F(t, y)|=h(t)|y(t)|, S(\tau) \subset L_{w}^{p}(a, b)$, $S\left(\tau^{+}\right) \subset L_{w}^{q}(a, b)$ for some $\lambda_{0} \in \mathbb{C}$ and $h(t) \in L_{w}^{p}(a, b)$ for some $p \geq 2$, $t \in[a, b)$. Then all solutions $\varphi(t, \lambda)$ of the Equation (1.1) are in $L_{w}^{p}(a, b)$ for all $\lambda \in \mathbb{C}$.

Corollary 4.5. Suppose that for some $\lambda_{0} \in \mathbb{C}, S(\tau) \subset L_{w}^{p}(0, b)$, $S\left(\tau^{+}\right) \subset L_{w}^{q}(a, b)$ and $k(t) \in L_{w}^{p}(a, b)$. Then all solutions of the equations $[\tau-\lambda w] \varphi=w k$ are in $L_{w}^{p}(a, b)$ for every complex number $\lambda \in \mathbb{C}$.

Next, for considering (4.1) with $0 \leq \sigma<1$, we have the following:

Theorem 4.6. Suppose that $F(t, y)$ satisfies (4.1) with $0 \leq \sigma<1$, $S(\tau) \cup S\left(\tau^{+}\right) \subset L_{w}^{\alpha}(a, b), \alpha \geq 2$ for some $\lambda_{0} \in \mathbb{C}$ and that

(i) $k(t) \in L_{w}^{\alpha}(a, b)$ for all $t \in[a, b)$,

(ii) $h(t) \in L_{w}^{\alpha /(\alpha-1-\sigma)}(a, b)$ for all $t \in[a, b)$.

Then all solutions $\varphi(t, \lambda)$ of the Equation (1.1) are in $L_{w}^{\alpha}(a, b), \alpha \geq 2$ for all $\lambda \in \mathbb{C}$.

Proof. For $0 \leq \sigma<1$, the proof is the same up to (1.1). In this case (4.5) becomes 


$$
\begin{aligned}
|\varphi(t, \lambda)| \leq & \sum_{j=1}^{n}\left(C_{j}+\left|\alpha_{j}(\lambda)\right|\right)\left|\varphi_{j}\left(t, \lambda_{0}\right)\right|+\left|\lambda-\lambda_{0}\right| \\
& \times\left.\sum_{j, k=1}^{n}\left|\xi^{j k}\right|\left|\varphi_{j}\left(t, \lambda_{0}\right)\right| \int_{a}^{t} \overline{\varphi_{k}^{+}\left(t, \lambda_{0}\right)}|h(s)| \varphi(s, \lambda)\right|^{\sigma} w(s) d s .
\end{aligned}
$$

Applying the Cauchy-Schwartz inequality to the integral in (4.8), we get

$$
\begin{aligned}
& \int_{0}^{t}\left|\overline{\varphi_{k}^{+}\left(s, \lambda_{0}\right)}\right||h(s)||\varphi(s, \lambda)|^{\sigma} w(s) d s \\
& \quad \leq\left(\int_{a}^{t}\left|\overline{\varphi_{k}^{+}\left(s, \lambda_{0}\right)}\right|^{\mu}|h(s)|^{\mu} w(s) d s\right)^{\frac{1}{\mu}}\left(\int_{a}^{t}|\varphi(s, \lambda)|^{\alpha} w(s) d s\right)^{\frac{\sigma}{\alpha}},
\end{aligned}
$$

where $\mu=\alpha /(\alpha-\sigma), \alpha \geq 2$. Since $\varphi_{k}^{+}\left(t, \lambda_{0}\right) \in L_{w}^{\alpha}(a, b)$ for some $\lambda_{0} \in \mathbb{C}, k=1,2, \ldots, n$ and $h(s) \in L_{w}^{\alpha /(\alpha-1-\sigma)} \cdot(a, b)$ by hypothesis, then we have $\varphi_{k}^{+}\left(t, \lambda_{0}\right)|h(t)| \in L_{w}^{\mu}(a, b)$, for some $\lambda_{0} \in \mathbb{C}, k=1,2, \ldots, n$. Using this fact and (4.9), we obtain

$$
\begin{aligned}
|\varphi(t, \lambda)| \leq & \sum_{j=1}^{n}\left(C_{j}+\left|\alpha_{j}(\lambda)\right|\right)\left|\varphi_{j}\left(t, \lambda_{0}\right)\right|+K_{0}\left|\lambda-\lambda_{0}\right| \\
& \times \sum_{j, k=1}^{n}\left|\xi^{j k}\right|\left|\varphi_{j}\left(t, \lambda_{0}\right)\right|\left(\int_{a}^{t}|\varphi(s, \lambda)|^{\alpha} w(s) d s\right)^{\frac{\sigma}{\alpha}},
\end{aligned}
$$

where $K_{0}=\left\|\varphi_{k}^{+}\left(t, \lambda_{0}\right) h(t)\right\|_{\mu},\|\cdot\|_{\mu}$ denotes the norm in $L_{w}^{\mu}(a, b)$. The inequality

$$
(u+v)^{\alpha} \leq 2^{(\alpha-1)}\left(u^{\alpha}+v^{\alpha}\right),
$$

implies that

$$
\begin{aligned}
|\varphi(t, \lambda)|^{\alpha} \leq & 2^{2(\alpha-1)} \sum_{j=1}^{n}\left(C_{j}^{\alpha}+\left|\alpha_{j}(\lambda)\right|^{\alpha}\right)\left|\varphi_{j}\left(t, \lambda_{0}\right)\right|^{\alpha}+2^{2(\alpha-1)} K_{0}^{\alpha} \\
& \times\left|\lambda-\lambda_{0}\right|^{\alpha} \sum_{j, k=1}^{n}\left|\xi^{j k}\right|^{\alpha}\left|\varphi_{j}\left(t, \lambda_{0}\right)\right|^{\alpha}\left(\int_{a}^{t}|\varphi(s, \lambda)|^{\alpha} w(s) d s\right)^{\sigma} .
\end{aligned}
$$


Setting $K_{1}=\int_{a}^{t}\left|\varphi_{j}\left(t, \lambda_{0}\right)\right|^{\alpha} w(s) d s \quad$ for some $\lambda_{0} \in \mathbb{C}, j=1, \ldots, n$ and integrating (4.11), we obtain

$$
\begin{aligned}
\int_{0}^{t}|\varphi(t, \lambda)|^{\alpha} w(s) d s \leq & K_{2}+2^{2(\alpha-1)} K_{0}^{\alpha}\left|\lambda-\lambda_{0}\right|^{\alpha} \sum_{j, k=1}^{n}\left|\xi^{j k}\right|^{\alpha} \\
& \times \int_{a}^{t}\left|\varphi_{j}\left(s, \lambda_{0}\right)\right|^{\alpha}\left[\left(\int_{a}^{s}|\varphi(x, \lambda)|^{\alpha} w(x) d x\right)^{\sigma}\right] w(s) d s,(4.12
\end{aligned}
$$

where $K_{2}=2^{2(\alpha-1)} \sum_{j=1}^{n}\left(C_{j}^{\alpha}+\left|\alpha_{j}(\lambda)\right|^{\alpha}\right) K_{1}$.

An application of Lemma 4.1 for $0 \leq \sigma<1$ and of Gronwall's inequality to (4.12) for $\sigma=1$ yields the result.

Theorem 4.7. Suppose that $F$ satisfies (4.1) with $0 \leq \sigma<1$, $S(\tau) \cup S\left(\tau^{+}\right) \subset L_{w}^{\alpha}(0, b) \cap L^{\infty}(a, b), \alpha \geq 2$ for some $\lambda_{0} \in \mathbb{C}$ and that

(i) $k(t) \in L_{w}^{\alpha}(a, b)$ for all $t \in[a, b)$,

(ii) $h(t) \in L_{w}^{p}(a, b)$ for some $p, 1 \leq p \leq 2 /(1-\sigma)$.

Then all solution $\varphi(t, \lambda)$ of the Equation (1.1) are in $L_{w}^{\alpha}(a, b) \cap L^{\infty}(a, b)$ for all $\lambda \in \mathbb{C}$.

Proof. Since $S(\tau) \cup S\left(\tau^{+}\right) \subset L_{w}^{\alpha}(a, b) \cap L^{\infty}(a, b)$ for some $\lambda_{0} \in \mathbb{C}$, then $\varphi_{j}\left(s, \lambda_{0}\right) \in L_{w}^{p}(a, b)$ and $\varphi_{k}^{+}\left(t, \lambda_{0}\right) \in L_{w}^{q}(a, b), j, k=1, \ldots, n$ for every $p, q \geq 2$ and for some $\lambda_{0} \in \mathbb{C}$.

First, suppose that $h(t) \in L_{w}^{p}(a, b)$ for some $p, 1 \leq p \leq 2$. Setting

$$
K_{0}=\left\|\varphi_{j}\left(t, \lambda_{0}\right)\right\|_{\infty} \text { and } K_{1}=\left\|\varphi_{k}^{+}\left(s, \lambda_{0}\right)\right\|_{\infty}, j, k=1,2, \ldots, n,
$$


we have from (3.4),

$$
\begin{aligned}
|\varphi(t, \lambda)| \leq & K_{0} \sum_{j=1}^{n}\left(C_{j}+\left|\alpha_{j}(\lambda)\right|\right)+K_{0} K_{1}\left|\lambda-\lambda_{0}\right| \\
& \times \sum_{j, k=1}^{n}\left|\xi^{j k}\right| \int_{a}^{t} h(s)|\varphi(s, \lambda)|^{\sigma} w(s) d s .
\end{aligned}
$$

Since $h(t) \in L_{w}^{p}(a, b)$ for some $p, 1 \leq p \leq 2$, then Lemma 4.1 together with Gronwall's inequality implies that $\varphi(t, \lambda) \in L^{\infty}(a, b)$ for all $\lambda \in \mathbb{C}$, there exist a positive constant $K_{2}$ such that

$$
|\varphi(t, \lambda)| \leq K_{2} \text { for all } \lambda \in \mathbb{C}, t \in[a, b) .
$$

From (4.8) and (4.14), we obtain

$$
|\varphi(t, \lambda)| \leq K_{0} \sum_{j=1}^{n}\left(C_{j}+\left|\alpha_{j}(\lambda)\right|+K_{3}\right)\left|\varphi_{j}\left(t, \lambda_{0}\right)\right|
$$

for an appropriate constant $K_{3}$. Since $\varphi_{j}\left(t, \lambda_{0}\right) \in L_{w}^{2}(a, b)$ for some $\lambda_{0} \in \mathbb{C}$, this proves $\varphi(t, \lambda) \in L_{w}^{p}(a, b)$ for all $\lambda \in \mathbb{C}, 1 \leq p \leq 2$.

Next, suppose that $h(t) \in L_{w}^{p}(a, b)$ for some $p, 2<p \leq 2 /(1-\sigma)$. Define $q \geq 2$ by

$$
\frac{1}{q}=\frac{\alpha-\sigma}{\alpha}-\frac{1}{p}
$$

(which is possible because of the restriction on $q$ ).

Thus $\varphi_{j}\left(t, \lambda_{0}\right) \varphi_{k}^{+}\left(s, \lambda_{0}\right) \in L_{w}^{q}(a, b) \quad$ and $\quad \varphi_{k}^{+}\left(s, \lambda_{0}\right) h(t) \in L_{w}^{\mu}(a, b)$, $\mu=\frac{\alpha}{\alpha-\sigma}, \alpha \geq 2 ; j, k=1, \ldots, n$. Repeating the same argument from (4.8) to (4.12) in the proof of Theorem 4.6, we obtain that $\varphi(t, \lambda) \in L_{w}^{\alpha}(a, b)$. Returning to (4.9), we find that the integral on the left-hand side is bounded, which implies by (4.8) that 


$$
|\varphi(t, \lambda)| \leq \sum_{j=1}^{n}\left(C_{j}+\left|\alpha_{j}(\lambda)\right|+K_{3}\right)\left|\varphi_{j}(t, \lambda 0)\right|
$$

for an appropriate constant $K_{3}$. Since $\varphi_{j}\left(t, \lambda_{0}\right) \in L^{\infty}(a, b)$ this completes the proof. We refer to [1], [7-9], and [17, 19] and for more details.

\section{References}

[1] R. P. Agarwal, A note on Grace and Lalli's paper, J. Math. Anal. Appl. 86 (1982), 471-475.

[2] D. E. Edmunds and W. D. Evans, Spectral Theory and Differential Operators, Oxford University Press, 1987.

[3] W. N. Everitt, The number of integrable-square solutions of products of differential expressions, Proc. of the Royal Soc. of Edinburgh 76A (1977), 215-226.

[4] W. N. Everitt and D. Race, Some remarks on linear ordinary quasi-differential expressions, Proc. London Math. Soc. 54(3) (1987), 300-320.

[5] H. Frentzen, Quasi-differential operators in $L^{p}$ spaces, Bulletin London Mathematical Soc. 31(3) (1999).

[6] S. R. Grace and B. S. Lalli, Asymptotic behavior of certain second order integrodifferential equations, J. Math. Anal. 76 (1980), 84-90.

[7] S. E. Ibrahim, Boundedness for solutions of general ordinary quasi-differential equations, Journal of the Egyptian Mathematical Society 2 (1994), 33-44.

[8] S. E. Ibrahim, On $L_{w}^{2}$-quasi derivatives for solutions of perturbed general quasidifferential equations, Czech. Math. J. 49(124) (1999), Praha, 877-890.

[9] S. E. Ibrahim, On the boundedness of solutions for products of quasi-integro differential equations, IJMMS 16(10) (2003), 639-659.

[10] S. E. Ibrahim, On the boundary conditions for products of general quasi-differential operators in direct sum spaces, IJAM 22(1) (2009), 55-87.

[11] S. E. Ibrahim, On the product of self-adjoint Sturm-Liouville differential operators in direct sum spaces, J. of Informatics and Mathematical Sciences 1 (2012), 93-109.

[12] S. E. Ibrahim, The regularly solvable operators in $L^{p}$ spaces, Fundamental Journal of Mathematics and Mathematical Sciences 2(1) (2015), 1-28.

[13] B. S. Lalli, On boundedness of solutions of certain second-order differential equations, J. of Mathematical Analysis and Applications 25 (1969), 182-188.

[14] J. S. W. Wong, Square integrable solutions of perturbed linear differential equations, Proc. Royal Soc. of Edinburgh 73A (1974/75), 251-254. 
[15] E. H. Yang, On asymptotic behavior of certain second order integro-differential equations, Proc. Amer. Math. Soc. 90 (1984), 271-276.

[16] E. H. Yang, Asymptotic behavior of certain second order integro-differential equations, J. Math. Anal. Appl. 106 (1985), 132-139.

[17] A. Zettl, Square integrable solutions of $L y=f(t, y)$, Proc. Amer. Math. Soc. 26 (1970), 635-639.

[18] A. Zettl, Perturbation of the limit circle case, Quart. J. Math. Oxford 26(3) (1975), 355-360.

[19] A. Zettl, The limit point and limit circle cases for polynomials in differential expressions, Proc. Royal Soc. of Edinburgh 72A (1975), 219-224.

[20] A. Zettl, Formally self-adjoint quasi-differential operators, Rocky Mountain Journal of Mathematics 5 (1975), 453-474. 